\title{
Baseline projections of energy and emissions in Asia
}

\author{
Geoffrey J. Blanford ${ }^{\mathrm{a}, *}$, Steven K. Rose ${ }^{\mathrm{a}}$, Massimo Tavoni ${ }^{\mathrm{b}}$ \\ a Electric Power Research Institute (EPRI), United States \\ ${ }^{\mathrm{b}}$ Fondazione Eni Enrico Mattei (FEEM), Italy
}

\section{A R T I C L E I N F O}

\section{Article history}

Received 25 October 2011

Received in revised form 17 August 2012

Accepted 20 August 2012

Available online 7 September 2012

\section{JEL classification:}

011

044

053

Q32

Q43

Keywords:

Integrated assessment modeling

Baseline scenarios

Emissions projections

Asia

\begin{abstract}
A B S T R A C T
This paper analyzes the projected development of energy systems in the Asia region in the hypothetical absence of future carbon policies. Baseline scenarios prepared by participating teams in the Asia Modeling Exercise are used to generate a comprehensive assessment of the key drivers of $\mathrm{CO}_{2}$ emissions for the next several decades, especially for China and India. We find a very wide range of projected emissions paths across the models and identify per capita income and energy intensity as the two major factors responsible for the variation. While the range of assumptions for growth in the former is roughly consistent with historical experience in other Asian economies, models foresee faster reductions in the latter with respect to those observed in neighboring countries at similar stages of economic development. On the other hand, there is a considerable agreement on the evolution of the energy technology mix, which is assumed to continue to be dominated by fossil fuels in the foreseeable future.
\end{abstract}

(c) 2012 Published by Elsevier B.V.

\section{Introduction}

A fundamental first step in the modeling and analysis of climate policy choices is the construction of a baseline scenario. This scenario reflects a projection, not a prediction or forecast, of a plausible evolution of energy and emissions in the absence of new greenhouse gas policies. Such a projection, in particular in terms of the scale of the energy system but also its technology mix, is essential as a basis for modeling scenarios in which policies are introduced. That is, the policy scenario outcomes can only be interpreted as conditional upon the corresponding baseline scenario. Because the energy-economy models used for an integrated assessment of climate policy are designed to measure the impacts of new policies, rather than model economic growth itself, the assumptions driving baseline projections are for the most part entirely exogenous. Naturally there is uncertainty about future development and $\mathrm{CO}_{2}$ emissions pathways, and models must make assumptions about growth, technology, and preferences that are both internally consistent and related in some way to historical trends. It is therefore essential to examine these assumptions and identify and analyze differences across models.

This paper provides an evaluation of baseline scenarios used by the energy modeling community for the Asian region. In terms of population size and economic growth, Asia is leading the world. China has already

\footnotetext{
* Corresponding author.
}

become the single largest emitter of $\mathrm{CO}_{2}$ in the world, and other developing countries, especially in South and Southeast Asia, are poised to enter stages of economic transition typically associated with a marked increase in energy consumption and associated emissions of greenhouse gases. Asia represents currently a third of global emissions and is projected by most models to reach half of the global total by mid-century. Results of coordinated modeling studies of climate policy scenarios have shown that the extent to which developing countries, especially rapidly emerging Asian countries, are involved in reducing $\mathrm{CO}_{2}$ emissions can significantly affect the feasibility and costs of achieving climate stabilization (see for example the EMF22 exercise, Clarke et al., 2009). Understanding the drivers of baseline growth for a major subset of the developing world is an important extension to this finding. The baseline pathway for emissions essentially scales the required abatement to meet a particular physical target, and thus is a major determinant of policy cost and feasibility.

Given the high level of inertia of the energy sector, on both the supply and demand sides, projecting the 'natural' evolution of the energy system, especially in fast growing economies such as China and India, is a major research task. Uncertainty is amplified for Asia for several reasons. First, these countries are characterized by very dynamic economies with high rates of growth. Second, the energy sector is also in a rather dynamic phase characterized by volatile energy prices, political instabilities in key exporting regions, and changes in technology and its applicability. Finally, policies for either national security, economic development or 
the protection of the environment are already being implemented in developing countries at a rather fast pace. The relative strength of these effects has been changing over time, and is the basis of the wide and changing range of projections, including those of international agencies such as the Energy Information Administration (EIA) and International Energy Agency (IEA).

The aim of this paper is to characterize baseline energy and emissions projections in the Asian region by comparing key aspects of these scenarios across a broad set of models. We use the results of the Asia Modeling Exercise (AME), a model comparison exercise with a focus on Asia (Calvin et al., 2012-this issue). Our methodology begins with a simple decomposition to identify key explanatory indicators, namely per capita income and energy intensity, and presents model results for these indicators in various forms, both in comparison with each other and with historical observations. Several insights emerge regarding the range of projections embodied in the study group and the implied extent to which future development in Asia will follow historical patterns. Finally, we examine projected changes in the technology mix in the energy system. We note that our methodology does not include statistical analysis, and we refrain from drawing any probabilistic inferences from the range of model output. Overall, we intend to illustrate what is, and what is not, of first-order importance in explaining the variation in baseline emissions projections and to evaluate projected changes in a historical context.

\section{Overview of baseline results}

We first present the main results of the AME baseline scenarios and provide a brief description of the range of participating model types. The AME data set comprised output from roughly 20 models with different structure, regional coverage, and focus of modeler interest and expertise. Many of the participating models are global in scope with regional disaggregation, but there are also models focused more narrowly on specific Asian countries. Models in the AME ensemble vary with respect to the representation of the economic activities, ranging from sectorally detailed computable general equilibrium models to aggregated growth models; technology detail on energy supply and end use, ranging from large-scale energy system models to compact representations of the energy sector; the degree of foresight and anticipation of future shocks, ranging from fully dynamic to myopic or simulation-based; and regional and time coverage. Thus, the data set provides us with a diverse group of approaches that well represents the state of the art of the modeling community.

All modeling teams were asked to simulate at least one baseline scenario. Importantly, no harmonization was stipulated for the scope of this comparison; that is, modelers were free to choose the key assumptions, thus consistency of views across models was not imposed. Since many of these assumptions, such as growth of population and the economy, as well as supply costs of energy carriers, have considerable bearing on the final output in terms of energy use and emissions, the AME data base provides ranges of projections that are not only model specific, but that also reflect the views of modelers on these exogenous drivers. Furthermore, no harmonization was required on the energy and climate policies to be included in the reference scenarios, such as energy technology standards, or market-based or regulatory mechanisms designed to reduce greenhouse gases. However, this information was collected in a companion questionnaire, which indicated that as a general rule the global integrated assessment models did not include specific policies in developing Asia, but that regional ones did on a more extensive scale. For example, in the case of China one regional model included current policies on energy efficiency improvement, development of new and renewable energy, and adjustment of economic structure which have major effects on the model output, effectively complicating the comparison with 'no policy' baselines.

A comprehensive report of baseline scenario outcomes through 2050 for economic output (gross domestic product, GDP), total primary energy ${ }^{1}$ (TPE), and energy-related $\mathrm{CO}_{2}$ emissions in major Asian countries is provided in a tabular format in Appendix 2. These outcomes are provided for reference; the illustrations and analysis in the body of the paper seek to elucidate the drivers for emissions projections in particular. In the paper we focus on insights specific to China and India, because of their global and regional importances and their wide representation as individual regions in participating models, although in some cases we are able to include results for other Asian countries. Additionally, although most integrated assessment models necessarily make projections into the distant future to capture long-term climate dynamics, we focus in this paper on the relatively near-term 2020 to 2030 horizon because of its greater relevance to policy discussions and more direct comparability with historical trends. As we shall see, this is sufficiently far in the future to introduce a significant range of uncertainty.

Fig. 1 shows the projections of fossil fuel and industrial $\mathrm{CO}_{2}$ emissions through 2030 for China and India. ${ }^{2}$ The first observation is that the range of projections across the models is undeniably large. By 2020, for both countries, projections differ by up to a factor of two, and by 2030 by up to a factor of three. Also observed is a surprisingly large discrepancy across models for the base year 2005 and the recent projection year 2010. This discrepancy, most likely caused by non-standardized data sources and conversion methodologies, is discussed in detail by Chaturvedi et al. (2012-this issue). For the current analysis, it is important to control for the different numerical starting points across models, so the comparisons that follow are based on indexed growth relative to 2005. Although the discrepancy appears large when comparing actual model results in Fig. 1, also shown by the shaded area is the range of indexed model projections with 2005 emissions normalized to the observed totals as reported by Oak Ridge National Laboratory (ORNL) in Boden et al. (2011). When the emissions paths are compared in indexed units, the range across models is reduced by only $10 \%$, suggesting that base year differences explain little of the variation in rates of future growth.

\section{Driver decomposition}

In order to assess the key indicators underlying emissions growth, we decompose emissions using the well known Kaya identity (an extension of the IPAT model of Ehrlich and Holdren, 1972):

$$
\text { Emissions }=\text { Population } \cdot \frac{G D P}{\text { person }} \cdot \frac{\text { Energy }}{G D P} \cdot \frac{\text { Emissions }}{\text { Energy }} .
$$

This identity allows for an intuitive identification of the main highlevel drivers of emission to four components related to population, per capita income or affluence, energy intensity of economic activity, and carbon intensity of energy. Because we apply the identity at the level of individual countries without further disaggregation (e.g. into sectors or household classes), no approximate attribution across additive sub-components of the ratios is necessary; the decomposition is straightforward and exact. Among these four components, it is known that affluence and energy intensity of GDP (the second and third terms) explain most of the variation in emissions levels. This is confirmed for the AME ensemble, as shown in Fig. 2 for the short term outlook in China. Here we show histograms for annual average rates of growth in each indicator for China between 2005 and 2020. Note that for this

\footnotetext{
${ }^{1}$ Note that primary energy is defined as direct equivalent, meaning that the contribution attributed to electric generation from nuclear and non-biomass renewable corresponds to the energy content of electric output, as opposed to primary equivalent, in which attribution to these technologies assumes a loss factor similar to fossil generation. While the primary equivalent method can distort physical units of energy supply, the direct equivalent method can give the misleading impression of declining or decelerating total primary energy when these technologies assume increasing shares of generation over time. However, this rarely occurs in the case of baseline scenarios.

${ }^{2}$ In general, individual models are not identified in the analysis in this paper. Further details on participating models can be found in Calvin et al. (2012-this issue).
} 

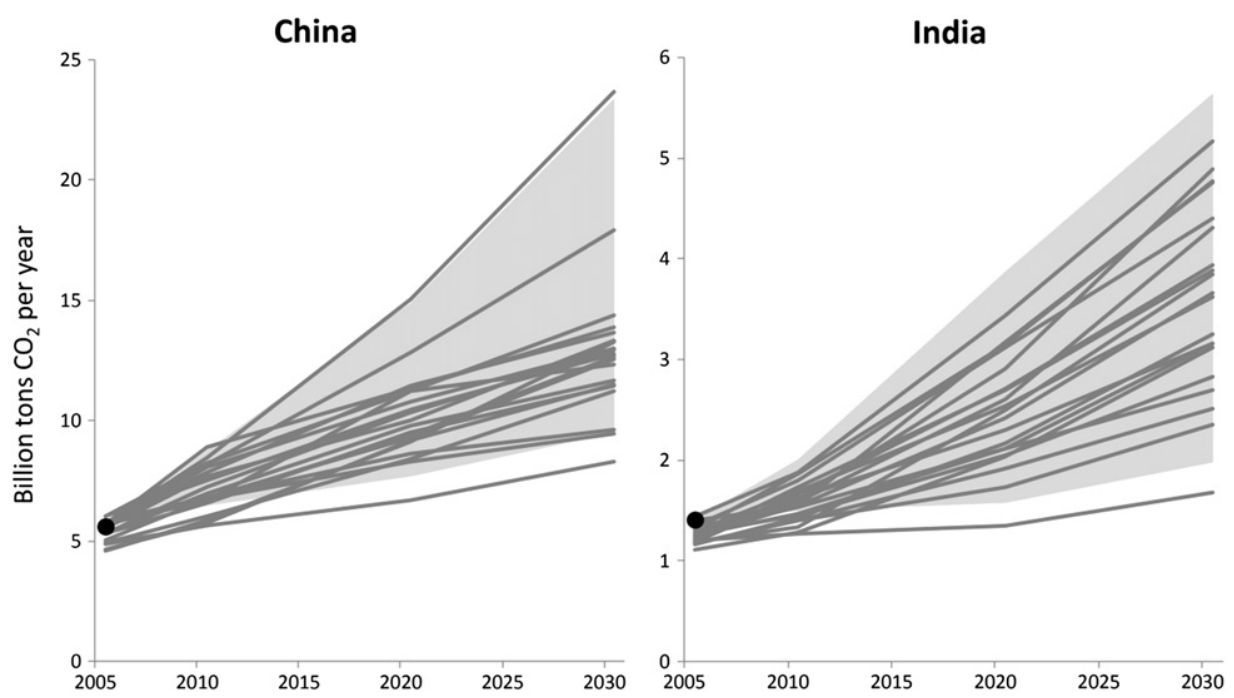

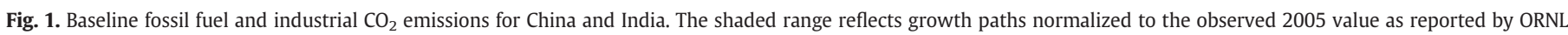
(black dot).

analysis we use primary energy net of traditional biomass as the energy metric in the third and fourth terms.

In this snapshot, population growth is essentially identical across models, despite the lack of harmonization, suggesting that most models rely on the same exogenous population projections, presumably those provided by the United Nations. There is also a rather good agreement on the carbon intensity of energy, a proxy for the composition of the energy mix, which is not surprising in a no-policy baseline scenario since it has historically changed very slowly. On the other hand, significant variations across models and much higher magnitudes of change are reported for both per capita income and energy intensity of GDP. Since the two have opposing signs, their relative strength will ultimately play the biggest role for determining $\mathrm{CO}_{2}$ emissions. We take a closer look at these two variables next.

\section{Historical comparison}

In the short term, the models in the AME data set project very rapid growth in per capita income for China between 2005 and 2020, with a median of $7 \%$ annual growth and a range from $5.1 \%$ to $9.2 \%$. While there are opinions as to the likelihood of outcomes in this range, one reasonable objective measure of validity is a comparison with historical data for similar countries at a similar stage of development. The countries against which we may compare China most naturally are its wealthier Asian neighbors, with some similarities in social and economic structures, portrayed over the epoch beginning when their respective real incomes matched China's income in 2005. To ensure a meaningful comparison across time and across countries, we use "real" incomes that have been normalized both for inflation and for the purchasing power of local currencies (see Appendix 1 for details). ${ }^{3}$

Fig. 3 shows the results of the historical comparison, with real incomes indexed to China's 2005 level in purchasing power equivalence as reported in the Penn World Table by Heston et al. (2011), roughly $\$ 4400$. $^{4}$ China's entire recent history is plotted, while for the other four countries we plot history for the 15-year period beginning the year real national income matched China's 2005 income. The range shown for China between 2005 and 2020 reflects the maximum and minimum average growth rates for these four historical comparison periods. The figure suggests that income growth in the model

\footnotetext{
${ }^{3}$ A similar exercise is described for the MERGE model in Blanford et al. (2009).

${ }^{4}$ A discussion of all data sources used, including a consideration of alternatives, is provided in Appendix 1.
}

projections for China is largely consistent with the mostly successful growth experience in other Asian countries. The projected range actually exceeds historical experience, but recent data suggests this shift may be appropriate as China is currently growing faster than any of its Asian predecessors. Moreover, the low end of the range of model projections implies a dramatic slowdown in the coming decade-e.g. growth on the order of 3\% per year between 2010 and 2020 after nearly $10 \%$ from 2000 to 2010 -an event that currently appears unlikely. Ultimately, the range across models for economic growth potential in the short term remains extremely wide, with a projected GDP in 2020 from twice to almost four times bigger than in 2005 given the very high growth rates of the Chinese economy.

A similar exercise can be done for energy intensity, again using the purchasing power equivalence for converting GDP, as shown in Fig. $4{ }^{5}$ In this case, China's neighbors all had lower energy intensity than China did in 2005 at the beginning of their respective income-calibrated epochs, but none realized any appreciable decline over the subsequent 15 years. However, the model projections for energy intensity in China are not consistent with this experience: all models foresee a decrease in energy intensity of at least $1 \%$ per year, some as high as nearly $6 \%$ per year, with a median of $2.7 \%$. Not only is the center of this range reflective of a rapid decline in energy use relative to economic activity, its breadth is substantial, implying again a factor of two in the overall scale of the energy system.

The projected trends of decline are indeed reminiscent of China's own history in recent decades. Beginning around 1980, China started from a very high level of energy intensity and was able to make substantial progress by transforming the planned economy through market reform, with the associated efficiency gains. Since 2000, this decreasing trend was dramatically reversed, mostly due to the build-up of large stocks of energy intensive capital in energy intensive industries such as cement and aluminum (although there is some question regarding the accuracy of reported energy statistics at the turn of the century). China's government set an aggressive goal of 20\% decline in energy intensity between 2005 and 2010, although recent data suggests this has not been met, as shown in Fig. 4. With growing domestic consumption potentially able to compensate for energy-intensive investments in the next several years, a declining trend could be regained, but the extent to which this is possible remains an open question. ${ }^{6}$ The energy

\footnotetext{
${ }^{5}$ Data sources for historical energy intensity are IEA (2007) and BP (2011).

${ }^{6}$ Calibrated models tend to implicitly incorporate this effect, postulating a crosscountry convergence to the same levels of intensity for the same levels of income in
} the short term. 

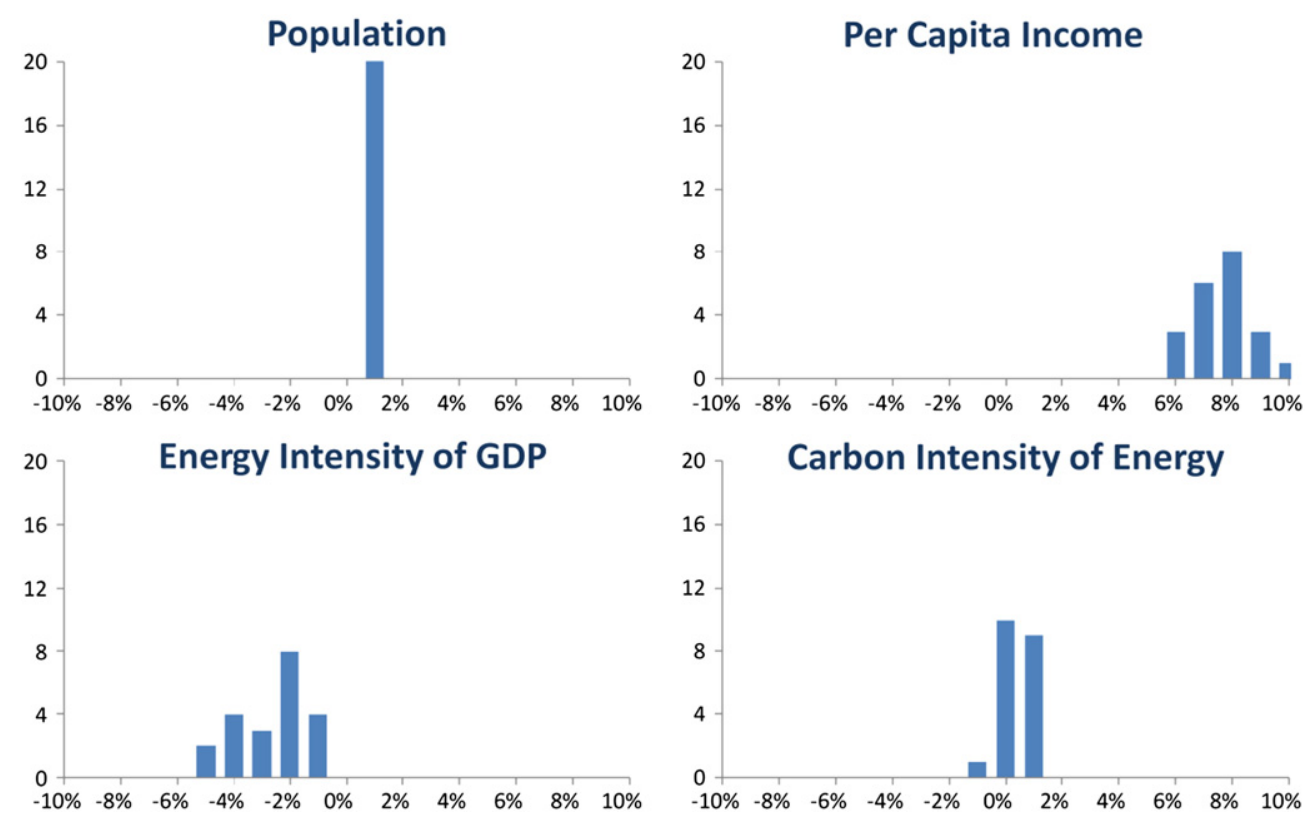

Fig. 2. Distribution across models in the AME baseline ensemble of average annual rates of change in China between 2005 and 2020 for each of the Kaya components.

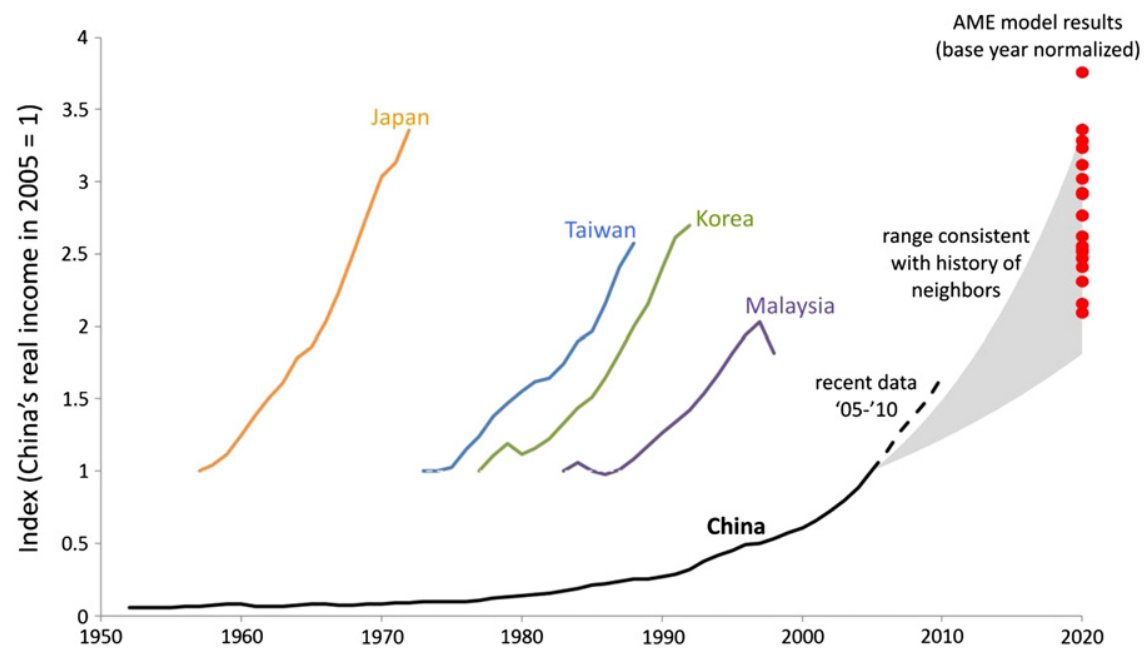

Fig. 3. Real per capita income: historical data for Asia and model projections for China in 2020. Data sources for conversion methodology are described in Appendix 1.

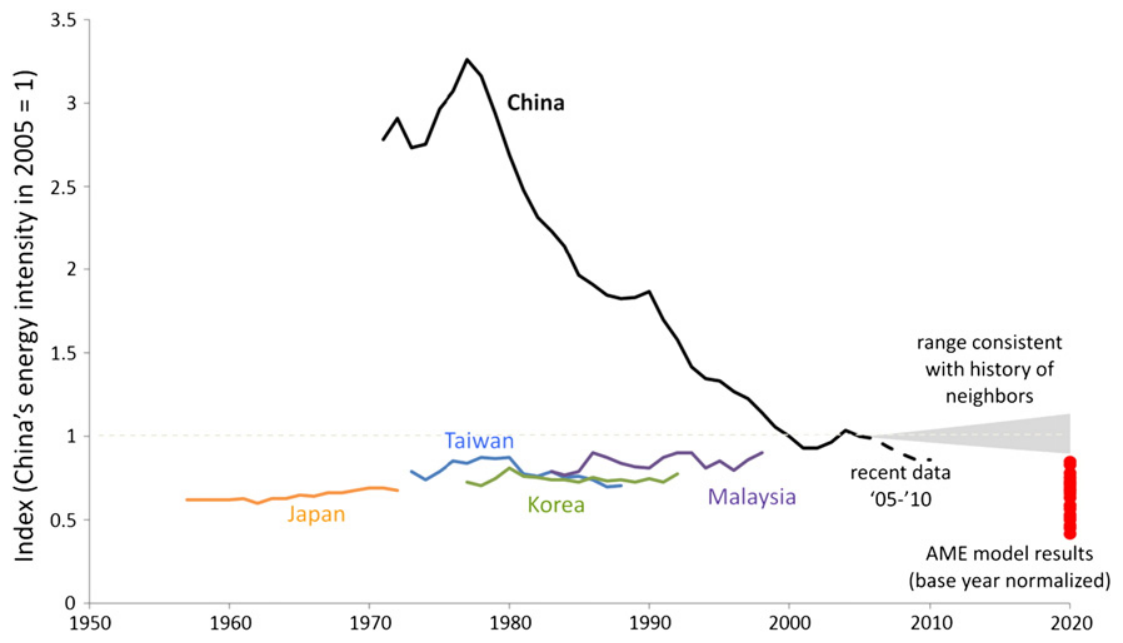

Fig. 4. Energy intensity of GDP: historical data for Asia and model projections for China in 2020. 
intensity trend highlighted by the AME models is nonetheless in line with the recently announced policy target for energy efficiency set forth in the latest five-year plan by China's government, which aims at a reduction of energy intensity of 16\% relative to 2010 by 2015.

\section{Cross-indicator comparison}

In order to further understand the relationship between economic growth and primary energy consumption growth, we present average annual rates of change in per capita income growth and energy intensity decline on a two-dimensional scatter plot. In addition to illustrating the ranges simultaneously and in relation to each other, this presentation can include diagonal isoquants for the rate of change in per capita energy consumption, the product of the two plotted variables. Because it encompasses both of the key drivers of emissions, per capita energy consumption (labeled PCE on the charts) is a useful metric for characterizing the overall emissions intensity of economic development. Fig. 5 shows the model output in this space for China and India over the period 2005 to 2020, as well as historical Asian data corresponding to the income-calibrated periods of comparison discussed above and depicted in Figs. 3 and 4. Also shown are projections for the 2020 time horizon (relative to 2005) from the EIA's International Energy Outlook (EIA, 2011) as an additional point of reference.

The observations highlighted above from the historical comparison with China are also evident in this figure: (i) the horizontal range of per capita income growth projections for China in the model ensemble is contained, with the exception of three high models, within the range spanned by the four historical examples, and (ii) the vertical range of energy intensity projections is well below that spanned by the historical sample. There are also several new insights. We can observe that the range provided by the models is very broad, with no apparent clustering around particular target scenarios, and in most cases projections depart significantly from recent history. Nearly all models suggest slower income growth than in the previous 15-year period, and a smaller majority project slower energy intensity decline. A slight correlation appears between the two dimensions, suggesting that the models with faster income growth assumptions also assume faster decline in energy intensity. The models cover a broad range of growth in per capita energy consumption, ranging from just over $1 \%$ to $6 \%$. Moving along the isoquant in the median range of $3.5 \%$ to $4 \%$, it is interesting to note that several models exhibit roughly equivalent per capita energy consumption growth with very different underlying circumstances. Some have relatively slow economic growth but also slow changes in energy intensity, while others have more rapid economic growth offset by more rapid intensity decline. While such scenarios will have similar emissions paths, they actually represent two quite distinct development paths for China.

Shifting our attention to India, we find that the AME ensemble projects income growth at a somewhat slower rate than for China, though still within the historical range of its Asian neighbors (although the historical data corresponds to a growth epoch during which incomes were higher than India's current income). Energy intensity decline rates are large and comparable to those projected for China, again much lower than those experienced by neighbors, which is notable given that India's energy intensity level is currently much lower than China's. Interestingly, the historical values for India in the recent past (1990 to 2005) are at the opposite extreme of the projection ranges compared to China. For India, most models project an acceleration in economic growth and energy intensity decline (with one exception in which energy intensity increases, similar to the historical pattern observed above), whereas for China economic growth and energy intensity (with a few exceptions) are decelerating. For both countries, per capita energy use is growing on the whole much more slowly than has been observed previously in Asia, and is growing more slowly in India than in China due to slower income growth. In one outlier projection, per capita energy use in India actually declines in the short term, a result that seems highly unlikely in a rapidly developing country, particularly given that we are measuring primary energy net of traditional biomass.

Model projections from 2005 to 2020 for three other Asian countries are presented in this format in Fig. 6, with the ranges for China and India represented roughly by shaded regions. While most models report Japan as a separate region, specific treatment of other Asian countries is less frequent. Projections for Japan are clearly much less dynamic than those for countries in earlier stages of development. Growth rates are in the range of $1 \%$ to $2 \%$, and more than half show a declining per capita energy use. ${ }^{7}$ While some models reporting data for Korea assume growth similar to that in Japan, the others envision a growth path for Korea more similar to a developing country in terms of rates of change. Indonesia, although represented in only a few models, is projected to follow growth patterns similar to, but generally slower than, those for India. Thailand, Malaysia, and Nepal are also projected in certain models but are not plotted here.

We look next at the relationship between per capita energy use and per capita income in Asian countries (Fig. 7). We now use absolute, rather than indexed, levels for the two indicators, although model projections are again normalized for base year consistency (that is, reported growth rates are applied to observed 2005 levels). Additionally, we show model projections for the reported ten-year time periods beyond 2020 to explore longer-term characteristics of the AME baseline scenarios (the 2020 projections corresponding to the previous charts are darker in color, while subsequent decades are lighter). We limit the per capita income scale to $\$ 50,000$ (2005 USD), so the chart excludes model projections in years for which income has grown beyond this level. However, nearly every model with a time horizon to 2100 projects that both China and India will reach this threshold before then, so the chart shows the full range of the projected per capita energy use for this income range. $^{8}$

This view affords a synopsis of the development patterns implied by model projections in relation to observed history. For the range of per capita incomes between $\$ 5000$ and $\$ 15,000$ (2005 USD), the history exhibits very consistent growth in per capita energy use, while different trends emerge for higher levels of income. Currently, China's per capita energy consumption is higher than in other countries at the same level of income (equivalent to the observed higher level of energy intensity shown in Fig. 4). Most models project that in the coming decades China will first align with the historical relationship, then fall below even the lowest energy-consuming nation in the sample to date, Japan. Still, a small number of models at the high end of the range depict a growth pattern in China that follows the historical relationship between income and per capita energy use. The solid red line shows a polynomial fit to the model projections for China, suggesting on average a lower slope on the relationship between income and per capita energy use than in observed Asian experience. However, the higher end of the range is roughly consistent with the experience of China's neighbors, and with its own recent history.

India's current per capita energy use is much lower than China's, but possibly more in line with the historical relationship, although again the historical data does not extend to India's lower income band. Like China, it is projected by the AME models to add per capita energy use more slowly on average as incomes rise than has been observed in other Asian countries. But since India's starting point is also lower, the emerging picture (with a few exceptions) describes a country with vastly lower per capita energy consumption than its neighbors across all income levels. The solid blue line shows a polynomial fit to the model projections for India, and following this path per capita energy use in India will not reach the current level in China until India reaches income levels similar to those in the OECD today. This result is striking and may raise

\footnotetext{
7 Projections for other developed countries such as the US and EU would fall in a similar region on the graph to Japan.

${ }^{8}$ It is necessary to include projections to ensure that the truncated income range is not populated disproportionately by fast-growing models.
} 
Per Capita Income Growth Rate

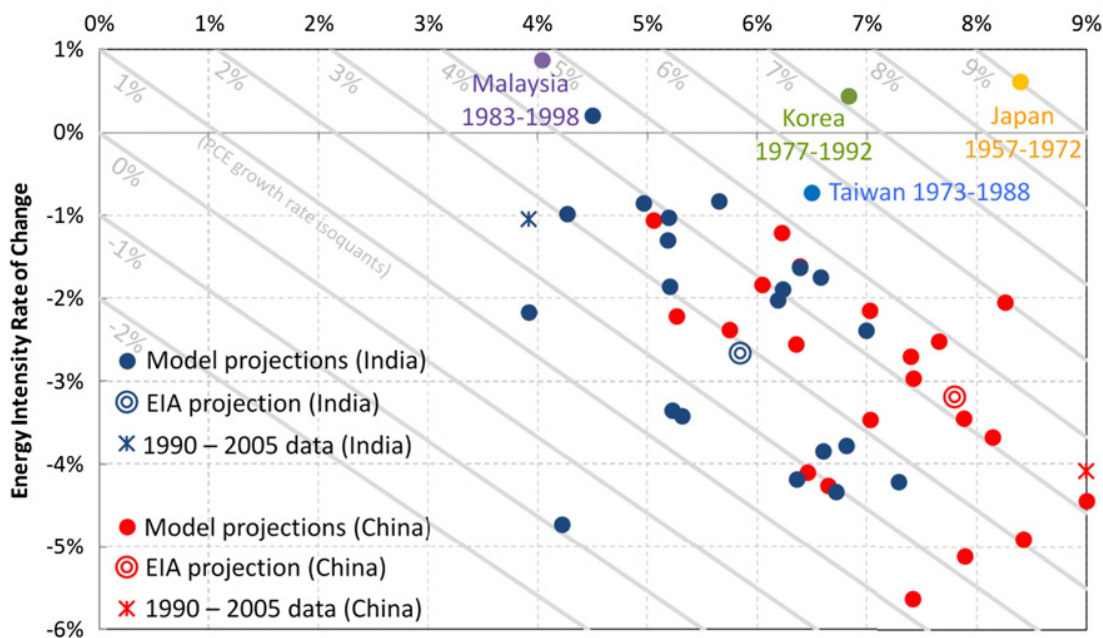

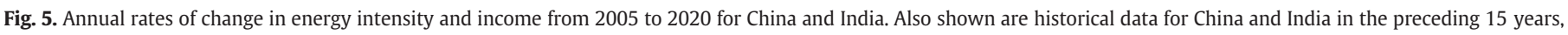
and for other Asian countries during the 15-year periods beginning when incomes matched China's 2005 income.

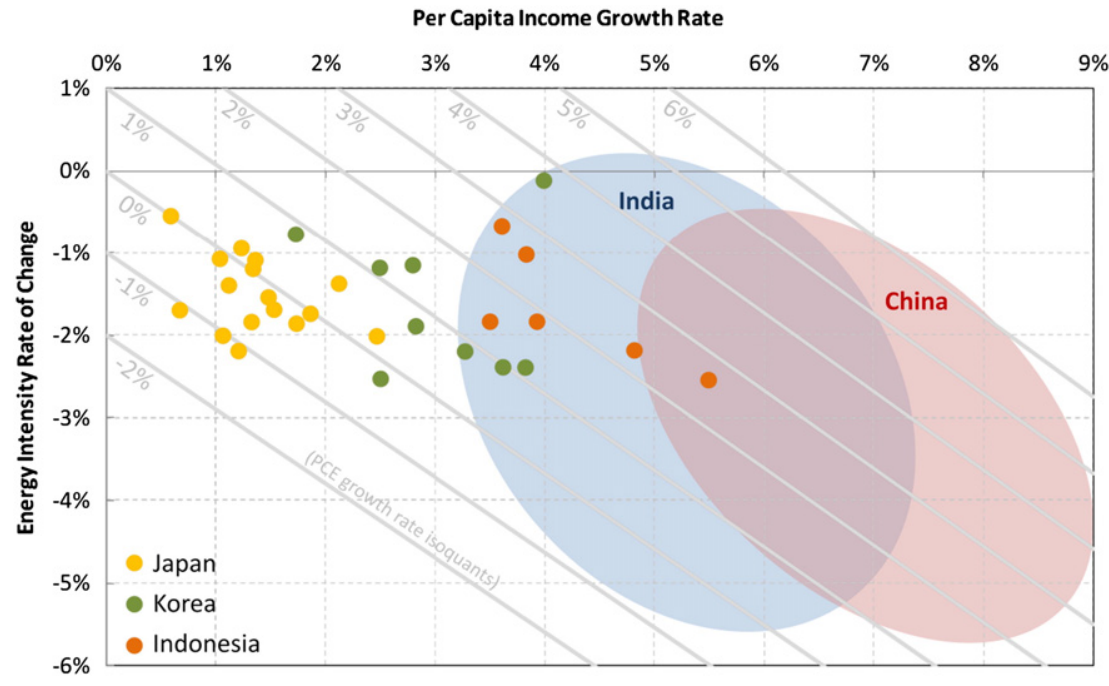

Fig. 6. Annual rates of change in energy intensity and income from 2005 to 2020 for Asia.

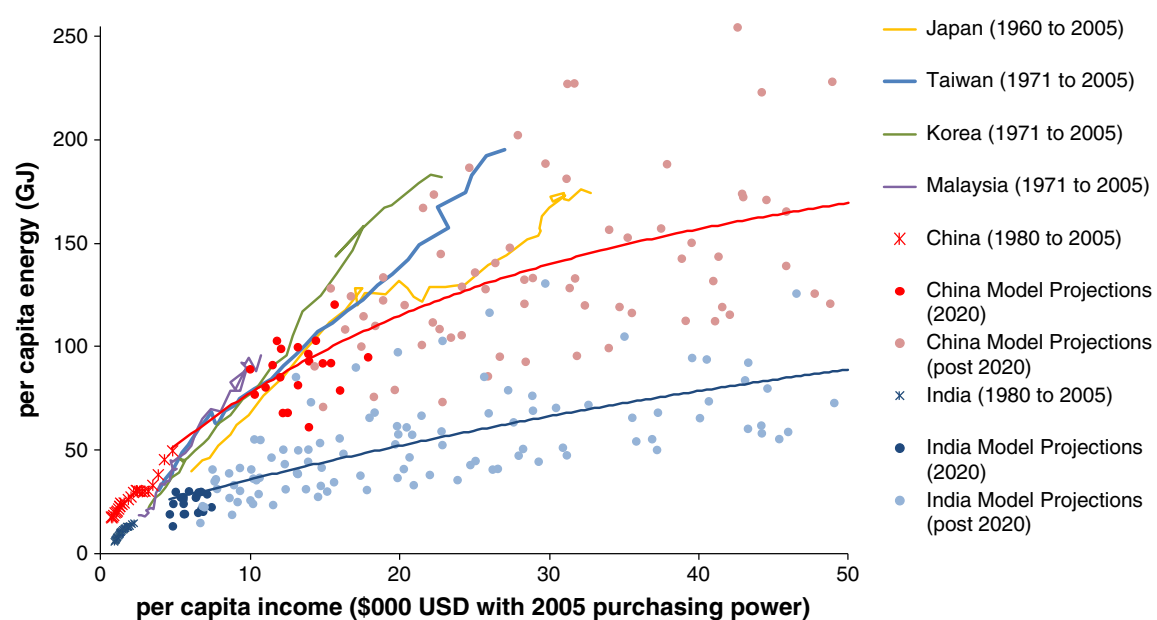

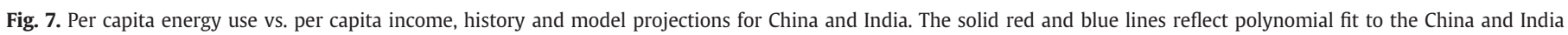
projections. 
questions about whether the projected rapid decline in energy intensity in the coming decades for India is indeed reasonable as it pursues its ambitious development goals.

An important caveat here is that income is hardly the only driver of per capita energy use, and the analysis has not controlled for other factors such as fuel prices, technology, and consumer preferences. Energy prices are probably on the whole higher now than during the sample period for the historical data, but subsidies also play an important role in China, India, and other developing Asian countries. Energy end-use technologies are undoubtedly becoming more efficient over time, but technical change also creates new demands for energy and expanded access to energy services. Unfortunately, the information in the AME data set on projected energy prices and costs and performance of technology was too sparse to conduct a formal investigation of these effects. Nonetheless, it is useful to explore the implications in aggregate of the role models project for energy in the development process.

\section{Income convergence}

Because of the central role played by income projections in the baseline scenario, and their importance for policy analysis, modelers may employ structural rules of varying levels of sophistication to motivate their assumptions. As we have illustrated, it is possible to use historical observations for both the country or region in question and other comparable regions to calibrate or evaluate near- and to a certain extent medium-term projections. In the longer run, these methods are not applicable, so growth rate projections may be based on top-down assumptions about total factor productivity growth, often combined either explicitly or implicitly with assumptions about the extent of income convergence across countries. In theory, increased connectedness and mobility of labor should reduce differences in income across countries, although there is inconclusive empirical evidence as to whether this has occurred in the past and whether it may be expected in the future. Nonetheless, complete or partial convergence over time is a useful concept for framing long-term growth projections. ${ }^{9}$ However, as we argue above, comparing incomes across countries for the purposes of assessing or positing convergence is most meaningful when done on a real purchasing power basis.

Using the indexed model projections for per capita income scaled by base year real income in China, for example, we can evaluate the ratio between this implied future real income and the projected income for the US. The results are shown in Fig. 8 for all models through 2100 . The wide range of projected relative incomes is due to a variation in both China's income and the US's income, but since the latter is universally assumed to grow more slowly, there is a movement toward convergence in all models. Still, there is very little agreement, with the ratio ranging from $30 \%$ to $80 \%$ in 2050 . Five models even exceed the "convergence threshold" before the end of the century, that is, their projections imply that China will become wealthier than the US on a per capita basis.

\section{Carbon Intensity and technology mix}

We now turn to the last component of the Kaya identity, carbon intensity of energy. As we have noted, there is a general agreement that average emissions per unit of primary energy will change a little along the baseline. In China, the rates of change in carbon intensity projected by most models from 2005 to 2020 are between $-0.5 \%$ and $0.5 \%$, with a few outliers projecting a faster decline. A variety of national policy measures designed to promote low-carbon technologies have been proposed in China, which are intended to be excluded from the

\footnotetext{
${ }^{9}$ Note that we do not intend to suggest that scenarios in which incomes converge are normatively preferable to those in which they don't. Rather we show descriptively the extent to which model projections in the AME baseline ensemble for China do or do not approach convergence.
}

baseline scenario, although it is unclear whether this is strictly true for all models in the AME data set. For models in which no new policies are included in the baseline, the prevailing economics favoring fossil fuel technologies, in particular coal-fired electric generation, remain in place. Although the carbon intensity of energy is unlikely to decline significantly in the absence of policy, the projected declines in energy intensity of GDP imply corresponding declines in the carbon intensity of GDP. In light of China's pledge under the Copenhagen Accord to reduce carbon intensity of its GDP in the range of $40-45 \%$ by 2020 relative to 2005 , it is interesting to ask whether this target is met in the AME baseline ensemble.

Fig. 9 shows a plot analogous to the previous charts with carbon intensity of energy on the horizontal axis instead of income. As before, diagonal isoquants may be drawn representing the rate of change in the product of carbon intensity of energy and energy intensity of GDP, namely carbon intensity of GDP. China's target reduction under the Copenhagen Accord amounts to a roughly 3.5\% to $4 \%$ annual rate of decline in the carbon intensity of GDP for the 15-year period, depicted on the chart. The figure shows that the AME baseline ensemble is roughly split in half by the Copenhagen target, with an approximately equal number of models suggesting that such a goal would and would not be met in the baseline. ${ }^{10}$ The question of whether the proposed policy would be binding or not is thus left unanswered by the ensemble of AME projections, although it is instructive to notice that the main source of disagreement across models is once again the projected changes in energy intensity of GDP, rather than those of carbon intensity of energy. In fact, nearly all the models that show the target being met actually have increased carbon intensity of energy, which is sufficiently offset by energy intensity decline.

Although per capita income and energy intensity projections vary substantially, resulting in very different scales for total energy demand, the modest projected changes in carbon intensity depicted in the previous chart for China suggest that the mix of fuels and technologies within the energy system is relatively stable. Fig. 10 shows the major components of projected electric generation for China through 2030. With a few exceptions, only relatively minor changes are observed in the mix: coal continues to dominate with a very large fraction (roughly 80\%) of power generation; hydroelectric share declines slowly, presumably as a result of the exhaustion of good sites; and nuclear expands slowly, not exceeding $10 \%$ by 2030 . A small number of models foresee rapid growth for either natural gas or renewables, particularly wind. Very similar results are seen in India and other regions with respect to the electricity mix. Finally, we note that there is also a general agreement that the share of electricity in primary energy will increase in the coming decades for both India and China, with electricity production growing faster than total primary energy by one percentage point or more. This trend is consistent with increasing electrification in the historical record for both industrialized and developing countries.

\section{Conclusions}

This paper has provided a multi-model assessment of the major drivers of $\mathrm{CO}_{2}$ emissions in Asia, with a specific focus on the large and fast-growing economies of China and India. Both energy use and emissions projections in the AME data set span a formidably large range, with scale varying by a factor of two or more even by 2020. Using the standard Kaya decomposition, we show that assumptions about changes in income and energy intensity are by far the most important determinants of the variation in energy consumption and emissions. Our historical comparison exercises have demonstrated that projected income

\footnotetext{
${ }^{10}$ Note that the EIA projection indicates that the target will be met, although it may be that the target itself was taken into consideration by the agency's projection. Also note that China did achieve a commensurate reduction in carbon intensity of GDP over the preceding 15 years, though as we have noted, this included a period of rapid energy intensity decline during the 1990s which may be difficult to reproduce.
} 


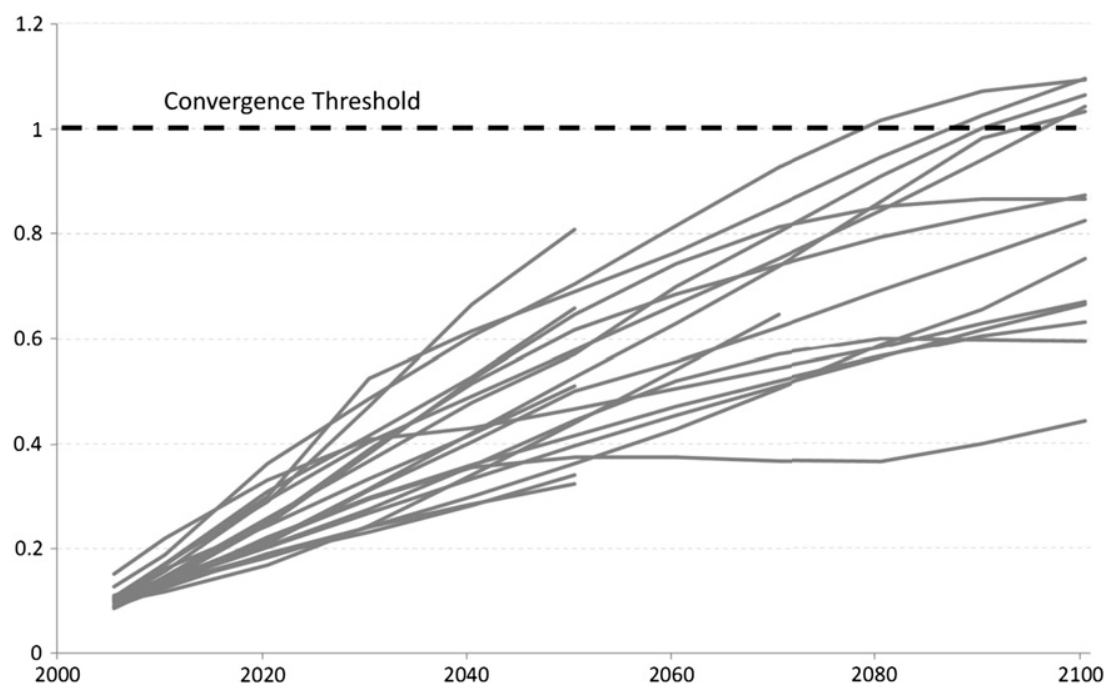

Fig. 8. Real income ratio between China and US in model projections.

growth is roughly consistent with recent Asian history, while projected declines in energy intensity are more aggressive than has been previously observed. For India in particular, these projections imply per capita energy use far below the historical norm throughout the development process. Lastly, the AME baseline ensemble exhibits a strong agreement that carbon intensity of energy, and the technology mix in general, is not projected to change significantly over the coming decades in the absence of new policies.

Both per capita income and energy intensity are tremendously dynamic in Asian countries at the current stages of development, and thus are particularly difficult to project. In this paper we have reported and compared model output for these indicators, but further work could be done to better understand the basis for projections made by individual modelers. For the models in this data set, per capita income is almost certainly an exogenous assumption, in the form of productivity improvements. On the other hand, energy intensity is partly driven by assumptions about autonomous energy efficiency change, but also by the response to relative price changes, as governed by the choice of various elasticity parameters, as well as the availability of alternative technological options. Thus, model harmonization is possible for incomes, but much more difficult for energy intensity.

Modeling energy intensity change is a research challenge. Energy demand is currently represented in most models at an aggregated scale, which can omit important substitution possibilities (as well as associated costs and barriers). For example, correctly representing fuel switching for household energy end-uses requires a micro-based understanding of the hurdle of changing behavior or replacing energy-using capital. Understanding the drivers for energy intensity change is also relevant for policy. Many countries are considering or actively implementing policies aimed at increasing energy efficiency as a way to hedge against volatile energy prices and profit from supposedly low-cost emissions mitigation opportunities. This paper has shown that integrated assessment models differ widely in their views of future Asian development of energy intensity, but all agree that it will decline more quickly than has been observed in history. An in-depth and formal analysis of what specifically underlies these decline projections was beyond the scope of this paper, and there remain gaps in the current reporting capability for coordinated model studies with respect to key explanatory data.

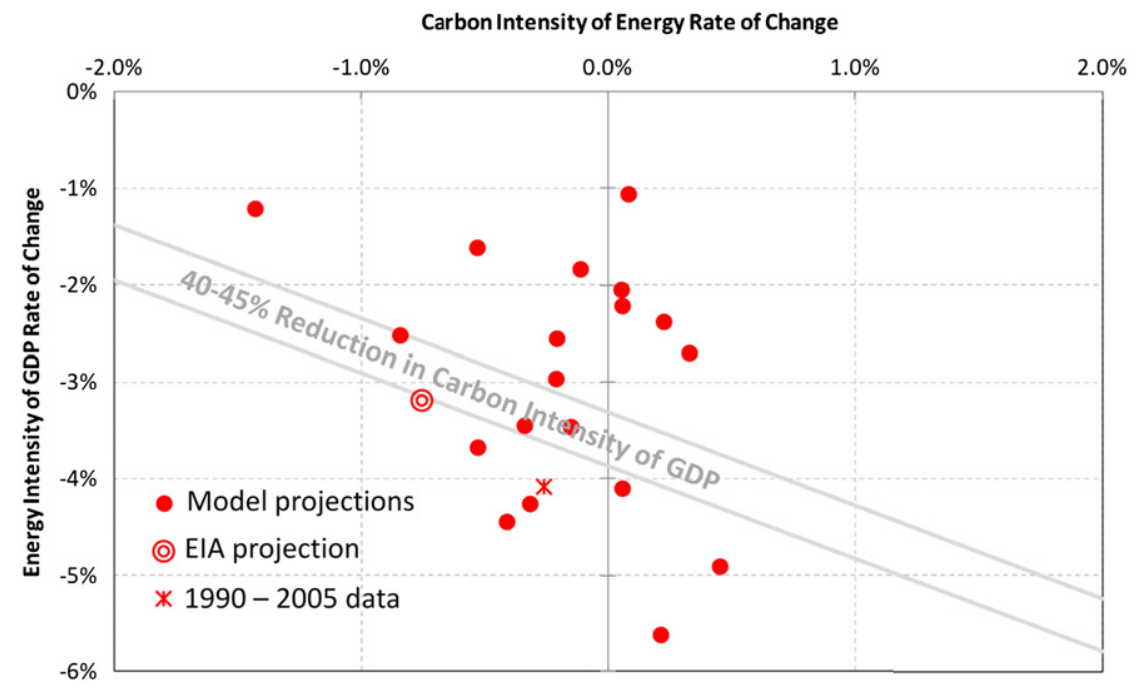

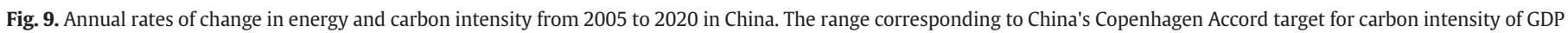
is also shown. 

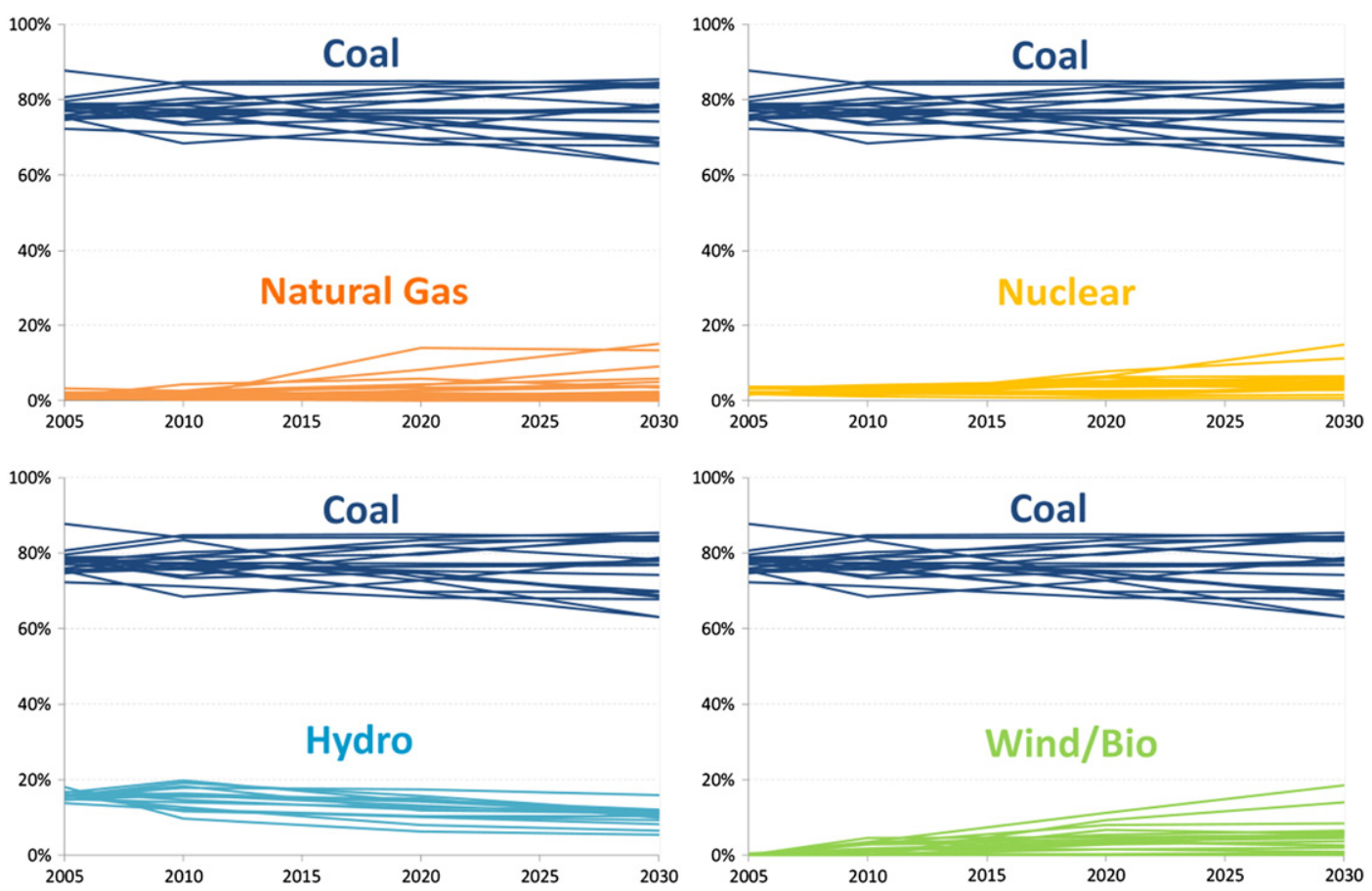

Fig. 10. Shares of electric generation in China.

A dedicated assessment of the modeling of energy intensity change might be a fruitful future avenue of research. In addition to better explaining the results of this study, such an assessment could facilitate an important advancement in the representation of energy systems in energy-economy models.

Three final concluding remarks are worthwhile. First, this analysis points to the general value of historical comparisons. Such comparisons can clearly identify similarities with or deviations from historical trends and development patterns and provide a richer context for discussions of the feasibility, even likelihood, of alternative futures. Second, the ranges reported here should not be regarded as a characterization of baseline uncertainty. The baseline projections in the AME ensemble represent a subset of baseline uncertainty, rather than a full characterization, which would entail a more coordinated consideration of input uncertainty, as well as differences in the model structure. Lastly, as noted in the Introduction section, interpreting climate policy scenarios is predicated upon an understanding of baselines. The models with lower projected $\mathrm{CO}_{2}$ emissions have lower climate policy costs and greater capacity for tighter targets. Across models with similar Asian population growth, we found that lower $\mathrm{CO}_{2}$ emissions baselines are signified primarily by rapid declines in regional energy intensity combined with lower income growth. Understanding and evaluating these baseline features are therefore critical to the integrated assessment of climate stabilization scenarios.

\section{Acknowledgments}

The authors are grateful to all the participants in the AME project, in particular Kate Calvin and Volker Krey, and to two anonymous reviewers for their helpful suggestions. We have attempted to ensure the accuracy of all reported model data but take responsibility for any remaining errors.

\section{Appendix A. Supplementary data}

Supplementary data to this article can be found online at http:// dx.doi.org/10.1016/j.eneco.2012.08.006.

\section{References}

Blanford, G.J., Richels, R.G., Rutherford, T.F., 2009. Revised emissions growth projections for China: why post-Kyoto climate policy must look east. In: Stavins, R., Aldy, J. (Eds.), Implementing Architectures for Agreement: Addressing Global Climate Change in the Post-Kyoto World. Cambridge University Press, Cambridge.

Boden, T.A., Marland, G., Andres, R.J., 2011. Global, Regional, and National Fossil-Fuel $\mathrm{CO}_{2}$ Emissions. Carbon Dioxide Information Analysis Center, Oak Ridge National Laboratory, U.S. Department of Energy, Oak Ridge, Tenn., U.S.A. http://dx.doi.org/ 10.3334/CDIAC/00001_V2011.

BP, 2011. Statistical Review of World Energy 2011. www.bp.com.

Calvin, K., et al., 2012. The role of Asia in mitigating climate change: Results from the Asia modeling exercise. Energy Econ. 34, S251-S260 (this issue).

Chaturvedi, V., et al., 2012. What are the starting points? Evaluating base-year assumptions in the Asia Modeling Exercise. Energy Econ 34, S261-S271 (this issue).

Clarke, L.E., Edmonds, J.A., Krey, V., Richels, R.G., Rose, S., Tavoni, M., 2009. International climate policy architectures: overview of the EMF22 international scenarios. Energy Econ. 31, S64-S81.

Ehrlich, P. Holdren, J., 1972. Review of The Closing Circle. Environment 24-39 (April).

EIA, 2011. International Energy Outlook. U.S. Energy Information Administration. September 2011.

Heston, A., Summers, R., Aten, B., 2011. Penn World Table Version 7.0. Center for International Comparisons of Production, Income and Prices at the University of Pennsylvania

IEA, 2007. World Energy Statistics 2007. International Energy Agency, Paris, France. 